\title{
A Study on Perpendicular Magnetic Anisotropy Cobalt Alloy Films Prepared by an Electroless Plating Method
}

\author{
Tetsuya OsakA*, Ichro Koma*, Yutaka Okabl:*, Hirosh MatslbarA*, Akihide WadA* \\ and Fumio GoTo**
}

\section{INTRODUCTION}

Recently, much effort has been spent on developing a perpendicular magnetic recording system, because of its high potential for high density magnetic recording. Various kinds of magnetic films prepared by physical vapor deposition methods have been studied for the perpendicular magnetic media. Only a few attempts (1,2), however, have been made so far to use the electroless plating method which provides better productivity. If perpendicular magnetic media could be obtained by wet processes, such as an electroless plating, the result would have great significance from the mass production point of view. Furthermore, it will realize a stable supply of the media needed to put that system into practical use.

This paper describes the electroless plated perpendicular anisotropy magnetic media and their recording characteristics.

\section{EXPERIMENTAL}

Electroless Co-Ni-Re-Mn-P alloy films were plated from an ammoniacal malonate-tartrate-succinate complex agents bath. A polished $\mathrm{Ni}-\mathrm{P}$ film chemically plated on $\mathrm{Al}$ alloy was used as a substrate. The Co alloy films were immediately plated $0.5 \mu \mathrm{m}$ thick on it without activation process. X-ray diffraction patterns and rocking curves for plated films were measured with an X-ray diffractometer with an $\mathrm{Fe}$ target. Magnetic properties of films were measured with a sensitive vibrating sample magnetometer and a torquemeter. The hysteresis loops were measured parallel $(/ /)$ and perpendicular $(\perp)$ to the film surface. Eight inch disks were prepared with the bath conditions selected. Disk conformation was the same as in the previous paper (2). The $0.05 \mu \mathrm{m}$ protective $\mathrm{SiO}_{2}$ overcoat was formed by a spin-coat method. Recording characteristics were evaluated with a $\mathrm{Mn}-\mathrm{Zn}$ ferrite ring head.

\section{RESULTS AND DISCUSSION}

The authors have already reported $(1,2)$ that the hcp structure $c$-axis of cobalt is easily oriented normal to the film plane with the addition of $\mathrm{MnSO}_{4}$ into the plating bath and that, nevertheless, the amount of $\mathrm{Mn}$ codeposition into the $\mathrm{Co}-\mathrm{Ni}-\mathrm{P}$ film is only about one weight percentage under optimum conditions. The uniform columnar structure of Co-Ni-Mn-P film obtained gives a high effective anisotropy magnetic field, $\mathrm{Hk}$, value, but the saturation magnetization, Ms, still remains larger. Ito et al. (3) reported a decrease in Ms value for the electroless Co-Ni-Mn-P film obtained by adding Re, but no perpendicular anisotropy filin on the disk substrate was able to be obtained because of bath instability, considerable change in magnetic properties by the substrate difference, and Hk-decrease with $\mathrm{Re}$ salt addition into the plating bath. A stable plating bath for complete anisotropy magnetic films has been developed by
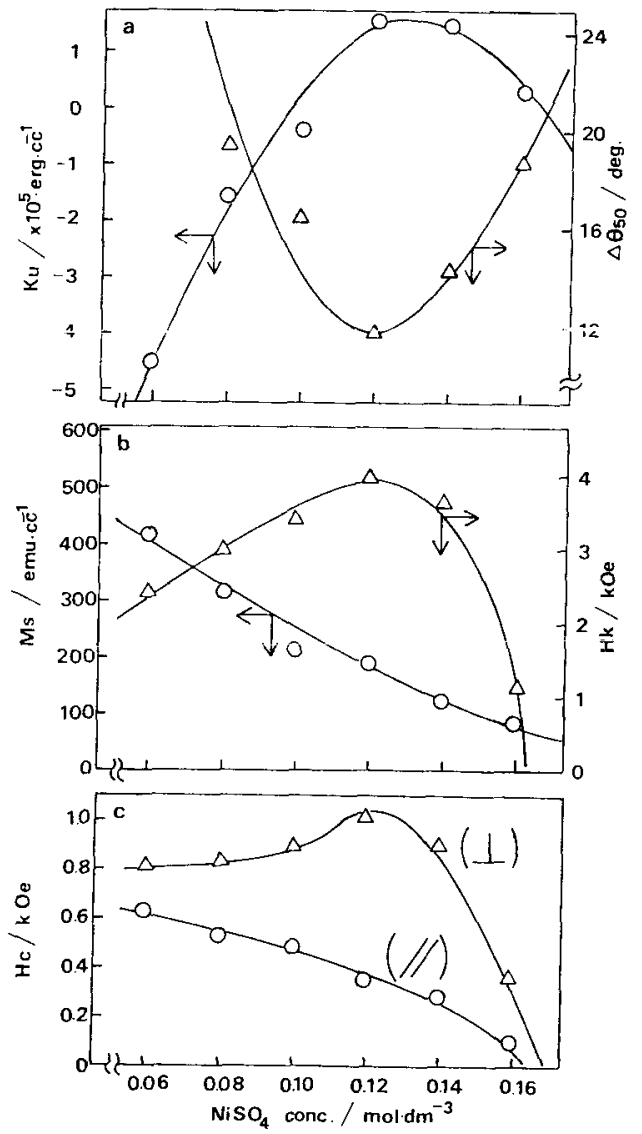

Fig. 1 Effect of $\mathrm{NiSO}_{4}$ concentration on magnetic properties and $\Delta \theta_{50}$

* Department of Applied Chemistry, WASEDA University (Okubo, Shinjuku-ku, Tokyo 160 JAPAN)

* Microelectronics Research Labs, NEC Corporation (Miyazaki, Miyamae-ku, Kawasaki 213 JAPAN) Key words; Electroless, Perpendicular magnetic recording, Magnetism, Cobalt alloy, Thin film 
using malonate-tartrate-succinate mixed complex agents for Co-Ni-Re-Mn-P films, based on extensive study of various complex agents and metal ion concentration effects on perpendicular anisotropy and bath stability. As a typical example, $\mathrm{Ni}(\mathrm{II})$ ion concentration effects on magnetic properties and half-maximum intensity peak breadth for the rocking curves of $\mathrm{Co}\left(\mathrm{OO}_{2}\right)$ plane, $\Delta \Theta_{50}$, are shown in Fig.1. The magnetic anisotropy energy, $\mathrm{Ku}$, increases with an increase in $\mathrm{Ni}(\mathrm{II})$ ion concentration and reaches a $1.6 \times 105 \mathrm{erg} / \mathrm{cc}$ maximum value at $0.12 \mathrm{~mole} / \mathrm{dm}^{3}$. The $\mathrm{Hk}$ value and difference in coercivities measured at perpendicular $(\perp)$ and parallel $(/ /)$, $\Delta \mathrm{Hc}=\mathrm{HC}(1)-\mathrm{HC}(/ /)$, become maximum at the same concentration range between 0.10 and $0.14 \mathrm{~mole} / \mathrm{dm}^{3}$ $\mathrm{Ni}(\mathrm{II})$ ion concentration. The $\Delta \Theta_{50}$ value also becomes minimum at the same $0.12 \mathrm{~mole} / \mathrm{dm}^{3}$ concentration range.

Eight inch disks were prepared by the improved plating bath. The Table shows disk and head characteristics, as well as read/write conditions. The spacing decrease from 0.25 to $0.20 \mu \mathrm{m}$ at $0.25 \mu \mathrm{m}$ magnetic film thickness improves the recording density at half maximum output voltage, D 50 , from 40 to 54 kFRPI, as shown in Fig.2a. When considering the $0.33 \mu \mathrm{m}$ head gap length, recording density $\mathrm{D} 50=54 \mathrm{kFRPI}$ using $0.20 \mu \mathrm{m}$ spacing is a relatively high recording density. The magnetic film thickness effect on recording properties is shown in Fig. $2 \mathrm{~b}$. Twice the magnetic film thickness at $0.20 \mu \mathrm{m}$ spacing surprizingly develops output voltage up to about four times, although accompanied by a slight decrease in recording density $\mathrm{D} 50=54$ to $51 \mathrm{kFRPI}$. The $30 \mathrm{~dB}$ SNR is also obtained for the medium. Such a high output voltage and a favorable SNR at high recording density under practically usable head-medium spacing value indicate a high plated disk potential for practical use as a perpendicular recording medium.
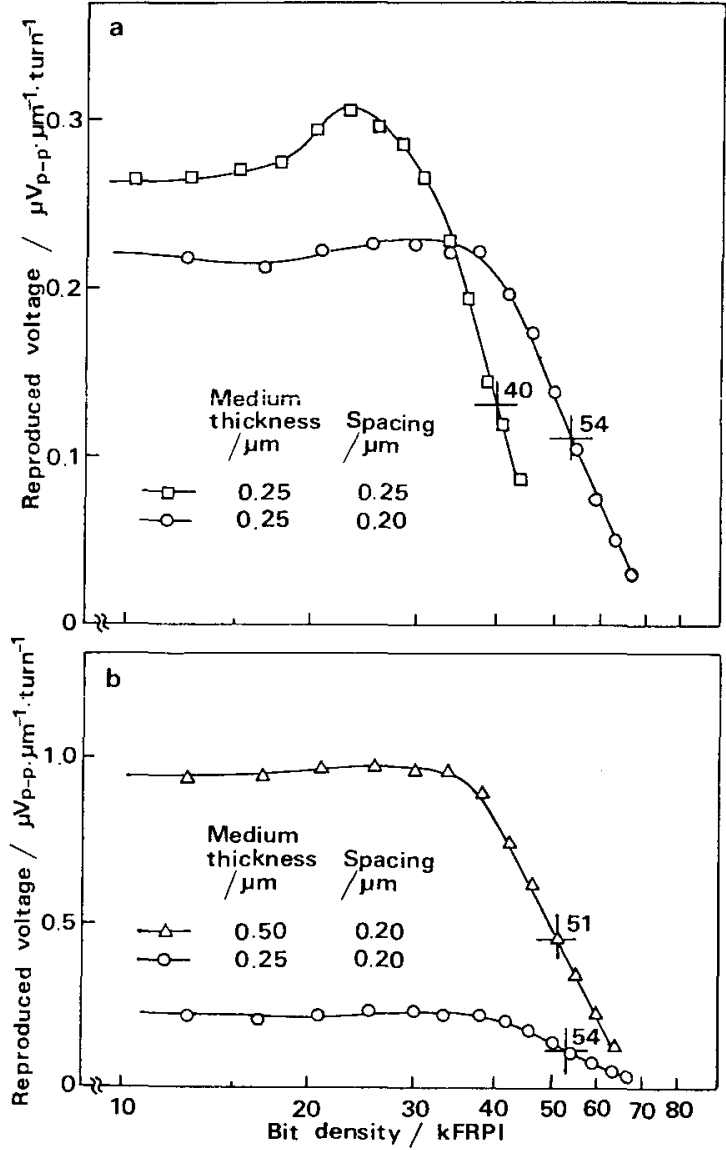

Fig.2 Reproduced voltage dependence on recording density
a) Spacing effect,
thickness effect

Table Disk and head characteristics

\begin{tabular}{|c|c|}
\hline \multicolumn{2}{|c|}{ DISK } \\
\hline MEDIUM MATERIAL & $\mathrm{Co}-\mathrm{Ni}-\mathrm{Re}-\mathrm{Mn}-\mathrm{P}$ \\
\hline MEDIUM THICKNESS & $0.25 \mu \mathrm{m} \quad 0.50 \mu \mathrm{m}$ \\
\hline OVERCOAT THICKNESS & $0.05 \mu \mathrm{m} \quad 0.05 \mu \mathrm{m}$ \\
\hline SUBSTRATE MATERIAL & Al alloy \\
\hline \multicolumn{2}{|l|}{ HEAD } \\
\hline CORE MATERIAL & \multirow{2}{*}{$\begin{array}{c}M n-2 n \text { ferrite } \\
0.33 \mu \mathrm{m}\end{array}$} \\
\hline GAP LENGTH & \\
\hline TRACK WIDTH & $155 \mu \mathrm{m}$ \\
\hline FLYING HEIGHT & $0.15 \mu \mathrm{m}$ \\
\hline & at $12 \mathrm{~m} / \mathrm{s}$ at $20 \mathrm{~m} / \mathrm{s}$ \\
\hline
\end{tabular}

\section{REFERENCES}

1) T. Osaka, F. Goto, N. Kasai and Y. Suganuma, Denki Kagaku, 49, 792 (1981).

2) T. Osaka, N. Kasai, I. Koiwa, F. Goto and Y. Suganuma, J. Electrochem. Soc., 130, 568 \& 790 (1983).

3) K. Ito, O. Takano and H. Matsuda, Abst. 66th Conf. Metal Fin. Soc. Japan, Oct. 1982, p.8.

(Recerved Jan. 14, 1984; Accepted Feb. 3, 1984) 